\title{
La metodología cualitativa en la demografía: una propuesta desde la fecundidad
}

\author{
Grisell Rodríguez Gómez*
}

\begin{abstract}
La pertinencia y validez de incorporar el enfoque metodológico cualitativo a las investigaciones demográficas, constituye el objetivo esencial de estas reflexiones. Se realiza inicialmente un análisis de las concepciones epistemológicas y metodológicas para el abordaje de la población desde la Demografía, repasando el objeto de estudio de la ciencia demográfica para realizar luego un breve recorrido sobre la evolución de la población e importantes teorías que han acompañado, descrito y explicado dicho proceso. Se aportan en consecuencia, elementos que validan la emergencia de lo cualitativo para las indagaciones en esta ciencia. Tomando por ejemplo, a la variable Fecundidad como objeto de estudio, se propone la aplicación de un enfoque teórico a partir de la delimitación de tres niveles entendiendo un nivel Macro Social, en el que se ubicaría a la Sociedad y un nivel Individual, entre estos dos extremos, estará mediando un nivel intermedio que podría denominarse instancia Meso Social y que a través de ella llegará la incidencia de lo social al individuo y su comportamiento, de este modo se validan la pertinencia de abordajes cualitativos y mixtos para explicar los comportamientos reproductivos. Finalmente se concluye que el posicionamiento epistemológico cualitativo, brinda herramientas y un modo de hacer, constructivista y explicativo, que estará aportando al cumplimiento del rol explicativo, predictivo y propositivo de la ciencia demográfica.
\end{abstract}

Palabras Claves: Metodología cualitativa. Demografía. Fecundidad.

\section{Introducción}

En el proceso de formación y consolidación de las ciencias sociales, se destacan dos perspectivas epistemológicas, que a su vez han dado lugar a determinadas corrientes metodológicas. Por una parte, es posible identificar paradigmas positivistas y neopositivistas que esencialmente conciben el mundo social como un conjunto de variables empíricas diferenciables, cuyas relaciones se expresan mediante lenguajes numéricos; son los que definen una corriente metodológica cuantitativa. Por otra parte, es posible identificar un paradigma humanístico-cualitativo, también denominado interpretativo, fenomenológico, naturalista o etnográfico. Este último se orienta a describir e interpretar fenómenos y se interesa por el estudio de los significados e intenciones de las conductas humanas. Este paradigma es

\footnotetext{
* Profesora del Centro de Estudios Demográficos (CEDEM) de la Universidad de La Habana desde el año 1995. Es licenciada en Psicología, Máster en Estudios de Población, Máster en Psicología Comunitaria y Doctora en Ciencias Económicas con especialidad en Demografía.
} 
el que determina una corriente metodológica cualitativa.

A lo largo de la historia de la ciencia, la relación cualitativo-cuantitativa ha tenido sus peculiaridades y han existido tendencias que hiperbolizan cada polo, a veces de manera excesiva. La concepción de ambas de manera distante, enfrentadas una a la otra, sin comunicación e interrelación, solamente obstaculiza el enriquecimiento del proceso de conocimiento de la realidad.

La delimitación de lo cualitativo en términos de investigación puede resultar compleja aún en estos tiempos, en los que parece que se han trascendido las fases iniciales de su introducción en el quehacer de las ciencias sociales. El uso de este término ha sido asumido de diversas maneras, ya sea desde una comprensión del mismo como forma diferente de hacer ciencia en relación al modelo positivista, o la reducción de este a su aspecto instrumental, manteniendo los paradigmas del método cuantitativo, (GONZÁLEZ, 1999) o la incorporación de un enfoque epistemológico, propio de su quehacer, pero alejándose de su utilidad y necesidad en la ciencia contemporánea.

Si bien es posible identificar momentos en los que era palpable un enfrentamiento entre modos de hacer cualitativos y cuantitativos, en el siglo actual se encuentra a sociólogos más centrados en la comprensión de las ventajas y desventajas, aportes y desaciertos, de los enfoques epistemológicos y metodológicos. Todo parece indicar que está teniendo prioridad la comprensión exhaustiva de los objetos del conocimiento. Las tan discutidas multi-, inter- y transdisciplinariedad están también acompañando esta concordancia e interrelación de enfoques cuali- y cuanti-. Se habla entonces con más frecuencia de saberes construidos a partir de la interrelación de lo cuantitativo y lo cualitativo.

El acercamiento de las ciencias a dichos enfoques o viceversa no ha sido, sin embargo, homogéneo, sino que, como también ocurre con los procesos que ellas estudian, este ha sido diferente en cuanto a la prontitud de su incorporación, los enfoques que priman y las resistencias encontradas. De ello, la Demografía, como ciencia que se ocupa del "estudio de los determinantes y las consecuencias del tamaño y la estructura de la población" (PRESTON, 1978, p. 34), no ha estado exenta y menos aún si se parte de que su comprensión y la historia de su surgimiento aparece entrelazada esencialmente a técnicas e instrumentos de corte cuantitativo. En consecuencia, cuando se consultan otras definiciones de Demografía, se encuentra que en las mismas se puntualiza lo cuantitativo como la perspectiva metodológica por excelencia para el análisis de su información (CELADE-UIECP, 1985).

El análisis de las concepciones epistemológicas y metodológicas para el abordaje de la población desde la Demografía que será realizado en lo sucesivo transitará por varios momentos. En primera instancia, un repaso al objeto de estudio de la ciencia demográfica y, a continuación, un breve recorrido sobre la evolución de la población e importantes teorías que han acompañado, descrito y explicado dicho proceso. La emergencia de lo cualitativo en esta ciencia, y una propuesta de aplicación a partir de la variable fecundidad, es finalmente la alternativa presentada.

\section{El estudio de la población desde la ciencia demográfica}

Resulta entonces imprescindible partir de la definición de qué entender por población y delimitar cuáles son los elementos que de esta población estudia la Demografía.

Inicialmente, se ha de partir del criterio de que es insustituible el término Demografía para acuñar a esta ciencia. Son varios los nuevos modos de nombrarla, con la intención de delimitarla y encausarla teórica y metodológicamente, entre los que se destacan: estudios de población o sociodemografía. En el primer caso, podría quedar delimitado de manera ambigua la intención del estudio y en el segundo caso parecería referirse a que la Demografía, como ciencia, se aísla o no comprende la sociedad en la que está insertada la propia población que ella estudia. En resumen, que no es preciso nombrarla de otro modo, sino más bien tratar de comprender en toda su esencia los propios fenómenos que ella estudia. 
En la determinación entonces de cuáles son los elementos de la población que estudia la Demografía, se arriba a la conclusión de que se ocupa de la estructura y dinámica de los componentes de las poblaciones humanas: fecundidad, mortalidad y migraciones. "La Demografía pretende describir estos tres aspectos de la población y encontrar sus determinantes y sus consecuencias (sociales, biológicas, económicas, etc.)" (WELTI et al, 1997, p. 17) Siendo así, la Demografía más allá de describir, de explicar los fenómenos, lo que pretende es ir a la búsqueda de causas y consecuencias, e incluso no es aventurado decir, que se dirige además a los procesos de construcción de los diferentes fenómenos.

Así pues, la Demografía sí precisa de enfoques epistemológicos y metodológicos que la auxilien en este empeño. Tienen cabida por ende en la Demografía enfoques cualitativos que la auxilien en la explicación de los elementos que rodean y condicionan el nacimiento, la muerte, la movilidad, así como el cúmulo de procesos que conforman las tres variables demográficas.

\section{Evolución de la población y de la teoría demográfica}

Ahora bien, la pertinencia de la incorporación a la Demografía de nuevos enfoques metodológicos tiene una explicación que se centra en el propio desarrollo teórico de la ciencia, que ha respondido en igual medida a la evolución de las poblaciones en lo que se refiere a los comportamientos de las variables demográficas.

Haciendo una rápida referencia históri$\mathrm{ca}$, es posible partir de que hasta antes de la Revolución Industrial, los niveles de mortalidad y de fecundidad de las poblaciones eran sumamente elevados, encontrándose entonces poblaciones con estructuras muy jóvenes, asociadas en un alto grado al bajo nivel de desarrollo en las sociedades de entonces. Sin embargo, desde mediados del siglo XVIII, con el advenimiento del desarrollo económico, que se irradió hacia los niveles y calidad de vida de la población, comenzó a disminuir la mortalidad de los pobladores de esas regiones, y ya para después de 1900 los avances médicos comenzaron a jugar un rol significativo. Posteriormente, en estos mismos países se inicia el descenso de los nacimientos paulatinamente. Estos procesos que estaban ocurriendo esencialmente en Europa y algunas zonas de América del Norte, comienzan a diseminarse hacia el resto de los países en desarrollo. De manera acelerada primero la disminución de la mortalidad, debido a la difusión del desarrollo industrial, pero sobre todo de los procedimientos y tecnología de salud, y, posteriormente, y de forma más lenta el descenso de los nacimientos.

El devenir histórico ha condicionado una disminución en el período que le ha tomado a cada región el tránsito de una a otra etapa de la transición. Mientras que a Europa, por ejemplo, disminuir la mortalidad le llevó casi medio siglo, América Latina muchos años después lo logró de manera más rápida, condicionado lógicamente por la transferencia de los adelantos e innovaciones en la medicina y en la tecnología en general, de conocimientos que fueron importados tras llevar algún tiempo consolidados, a la par de la difusión de patrones reproductivos.

Mientras tanto, el interés por estudiar y comprender los procesos de entradas y salidas de efectivos a las poblaciones, considerando sus niveles de movilidad y reproducción, ha llevado a los demógrafos a debates en torno a las teorías que están explicando muchos de estos fenómenos. Los postulados teóricos en el interior de la Demografía han estado acompañados del devenir de los procesos demográficos, en la misma medida en que ellos comienzan a ser estudiados. La fecundidad, la mortalidad y las migraciones, de manera independiente, han sido y están siendo explicadas de manera indistinta desde sus factores condicionantes hasta las delimitaciones de tendencias en sus comportamientos.

A partir de la evolución de las poblaciones y su interrelación con el desarrollo económico, algunos científicos describen los procesos de tránsito en las variables demográficas -mortalidad, fecundidad y migraciones, delimitando etapas que explican cambios en el interior de las mismas. 
Dichas teorías se desarrollan desde las experiencias históricas en el contexto europeo, definiéndose estados de descenso de los indicadores de la fecundidad y mortalidad y estableciendo vínculos con el desarrollo económico y social. A la par, han posibilitado anticipar el futuro demográfico de las regiones que se encuentran en las fases tempranas de la transición.

Los primeros enunciados sobre dicho tema fueron realizados por el francés Adolphe Laundry en 1909 y 1934, quien estudia los cambios globales en la reproducción de la población identificando tres 'regímenes' o estados de la población: el primitivo, el intermedio y el moderno. Establece entonces que es la productividad del trabajo, el factor económico, el que define dichos regímenes.

W. S. Thompson, por su parte y paralelamente en el tiempo, desarrolló una teoría partiendo de las experiencias de estas regiones. Agrupó así, las naciones en tres, de acuerdo a sus niveles de mortalidad y fecundidad, distinguió entonces a los países en tres fases: primeramente aquellos con un rápido descenso de las tasas de mortalidad $\mathrm{y}$ fecundidad $\mathrm{y}$, por ende, un descenso acelerado de las tasas de crecimiento, en segundo lugar, los países en los que van descendiendo los niveles de mortalidad y fecundidad por grupos sociales y en los que las tasas de mortalidad descienden tan o más rápido que las de natalidad, produciendo un crecimiento estable o ligeramente ascendente y, en tercer lugar, los países en los que ambos indicadores están menos controlados y no sujetos a limitaciones. A cada uno de estos estados los denominó correspondientemente: estacionario, de crecimiento efectivo y de crecimiento potencial. Esta descripción responde al nombre de Evolución Demográfica.

En el año 1945, Frank Notestein, también a partir de la experiencia europea, estableció un vínculo entre el proceso de modernización y los indicadores demográficos, definiendo tres estados en su Teoría de la Transición Demográfica. Parte del supuesto de que se produce inicialmente el descenso de la mortalidad, mientras que la fecundidad responde más lentamente a dicha modernización. Finalmente sí comienza dicho descenso, producto de la regulación consciente del tamaño de la descendencia y por la interrelación de factores como la urbanización y la modificación de aspiraciones y proyectos de vida de los individuos.

A partir de dichas teorías y sus contribuciones posteriores, se han venido describiendo los procesos de cambios en estos indicadores en las diferentes regiones.

La teoría transicional, por su parte, ha recibido diversas críticas referidas a la no directa determinación entre desarrollo económico y social con los cambios en dichas variables y provoca interrogantes en torno a cuáles son las condiciones bajo las que muchas regiones están alcanzando, en ausencia de un desarrollo económico y social estable, bajas tasas de mortalidad y natalidad y un ritmo de crecimiento cada vez más moderado. Sobre ello, aún le queda a la Demografía un camino por recorrer en la búsqueda de elementos de carácter teórico que aporten explicaciones.

Otra crítica sobre dicha teoría está referida a que en su esencia "posee un carácter positivista, dado que parte de las manifestaciones externas de los cambios en la reproducción (...), sin ofrecer una explicación de estos cambios" (HERNÁNDEZ, 1986, p.10).

Una tercera crítica que se le ha formulado consiste en señalar que la teoría en sí misma sólo formula la explicación de los cambios en los niveles de la fecundidad y la mortalidad, $y$, en consecuencia, el paso de una etapa a la siguiente, a partir sólo de la experiencia empírica de los países considerados desarrollados en el momento de haber sido enunciada y, por consiguiente, no recoge la gran diversidad de situaciones particulares alrededor de las poblaciones que iniciaron y completaron el proceso después de su aparición como modelo teórico explicativo, como tampoco toma en cuenta dentro de su explicación teórica la variable migración.

De la misma manera, deja un vacío en cuanto al umbral de desarrollo que es necesario alcanzar para que dé comienzo un proceso de transición demográfica, así como tampoco ofrece mucha luz en cuanto al tempo de todo el proceso de transición y a la conexión entre los fenómenos económicos y los cambios entre las etapas. 
Durante algo más de medio siglo, ha sido esta la teoría que ha conducido la explicación de los cambios en las variables demográficas en diversas regiones del mundo y ha ido ganando en la diversidad de elementos explicativos de los cambios demográficos que se han ido sucediendo, incluyendo el abordaje de las particularidades propias de las poblaciones que están aún en plena transición demográfica. Paralelamente, el propio desarrollo de la Demografía, unido a su interrelación con otras ciencias, condicionó la aparición de otras explicaciones teóricas que contribuyeron a profundizar el conocimiento sobre los factores que actúan en las propias trayectorias demográficas.

Sin embargo, pasada la segunda mitad del siglo XX, los demógrafos se encontraron de cara a una nueva situación: un grupo de países del continente europeo ya han alcanzado bajos niveles en las variables mortalidad y fecundidad, han estabilizado los valores de dichos indicadores y están mostrando otros elementos de carácter cualitativo que acompañan la evolución y comportamiento de sus poblaciones. Surge entonces la pregunta: ¿bajo qué postulados teóricos han de ser entendidos estos procesos demográficos? ¿Es suficiente lo enunciado en la teoría de la Transición Demográfica? ¿Qué pasará en el resto de los países y regiones que se encuentran en los umbrales del fin de su transición demográfica?

En estas circunstancias los investigadores europeos Dirk J. van de Kaa y R. Lesthaeghe iniciaron algunas consideraciones teóricas considerando, al igual que sus antecesores, las situaciones concretas por las que transitaban en este momento las regiones europeas e industrializadas y enunciaron una continuidad de la primera transición demográfica: "El nuevo estado en la historia demográfica europea podría llamarse: 'Segunda Transición Demográfica'” (VAN DE KAA, 1987, p. 4).

A partir de la década de los 80 s del pasado siglo, se comienzan a proponer ideas que confluyen hacia la delimitación de postulados - desde la empiria hasta algunos niveles de generalización- que conforman la llamada 'Teoría de la Segunda Transición demográfica'. Esta se refiere a la descripción e interpretación de los comportamientos de las variables demográficas en el contexto de las sociedades industrializadas de hoy, que culminaron su transición demográfica.

Se parte del hecho de que la situación demográfica actual en las regiones europeas está estrechamente vinculada con el funcionamiento de la individualidad en estas rápidamente cambiantes sociedades. Como consecuencia, casi todos los estudiosos de esta particularidad, coinciden en que las características demográficas encontradas en estas regiones son reflejo de un profundo cambio en las normas y actitudes de las sociedades y de los individuos. Los atributos más importantes de esta segunda transición son que las tasas de fecundidad se mantienen excepcionalmente bajas, la individualidad pasa a tener un muy importante rol, destacándose la autorrealización por encima de la preocupación por atenerse a patrones y normas de conducta tradicionales (COLEMAN, 2005).

Aún cuando se mantiene, al igual que en la primera transición demográfica, un estrecho vínculo con el contexto socioeconómico, como condicionante esencial de los niveles en las variables demográficas, se aprecia una diferencia esencial con relación a esta: se trasciende en el análisis desde un enfoque centrado en los valores cuantitativos de las variables fecundidad y mortalidad hacia la incorporación de los elementos cualitativos que las describen.

Adicionalmente, la variable migración asume un importante rol en condiciones en las que la fecundidad se mantiene sostenida en niveles inferiores al reemplazo. Se prevé a esta variable como la encargada de equilibrar el desequilibrio que se produce en la estructura por edades de la población.

En resumen, la segunda transición demográfica se está refiriendo a explicar que aquellas sociedades en las cuales se ha concluido la primera transición y se han alcanzado, por ende, niveles muy bajos de las variables mortalidad y fecundidad, sostenidos en el tiempo, se encuentran ante un proceso en el cual ya no se continuarán modificando sustancialmente estas variables 
en lo referido a sus niveles. Se comenzará a prestar atención en mayor grado a características asociadas a la cualidad que acompaña a dichas variables, entiéndase como tal modificaciones en patrones y normas de comportamiento con respecto a la sexualidad, a la maternidad, a la formación de parejas, a la familia, etc. que estarán incidiendo en indicadores de las variables.

Muchos de estos elementos están condicionando que aparezcan variaciones en la secuencia de eventos en la formación de la familia. En consecuencia, parece que no existe un claro orden de sucesión de los acontecimientos vitales adultez-matrimonionacimiento de hijos. Se están consolidando patrones en los que la unión consensual antecede al matrimonio, el nacimiento de los hijos se produce en ocasiones fuera del matrimonio, en condiciones de soltería o en uniones, el divorcio condiciona la formación de nuevas uniones y el nacimiento de nuevos hijos, y el modelo de la familia varía en correspondencia. Paralelamente, han sido identificados algunos aspectos relativos a los diferenciales de la mortalidad, así como de manera significativa a la importancia que reviste la migración internacional.

Esta llamada segunda transición demográfica, pone su énfasis de manera significativa en cambios relativos al comportamiento de los individuos frente a su reproducción, luego de sostenidos valores en los indicadores y del tránsito de las variables mortalidad y fecundidad hacia niveles bajos. La principal característica demográfica en esta etapa es su efecto sobre las estructuras por edades, al elevarse la proporción de personas en edades avanzadas, encontrándose las sociedades ante un acentuado envejecimiento de la población al que hacerle frente.

Lo más significativo de este proceso transicional desde las diferentes aproximaciones teóricas es asumirlo como un continuo, en el cual la última etapa de la transición demográfica sienta el precedente para que dichas sociedades postransicionales, una vez alcanzados determinados patrones de comportamiento, propicien, dentro de su propio desarrollo social, que aparezcan o se profundicen particularidades en sus actitudes ante la reproducción, la formación de familias, entre otras.

Al igual que ocurrió históricamente con la transición de las variables mortalidad y fecundidad, que no se produjo simultáneamente en el tiempo en las diferentes sociedades, poseyendo en cada región y país sus propias peculiaridades, estaría ocurriendo en la actualidad con la llamada segunda transición. Esta depende, en mucho mayor grado, de los patrones sociales y culturales de cada región y las sistematizaciones hasta ahora realizadas son, al igual que a principios del pasado siglo, a partir de la experiencia de los contextos europeos industrializados.

Pero existe también otro elemento de singular relevancia y que llama la atención sobre la búsqueda de explicaciones de los comportamientos demográficos. $Y$ se refiere a la mirada que está dirigiendo la comunidad de demógrafos hoy hacia los pequeños espacios, hacia las pequeñas poblaciones. Puesto que la Demografía ha sido pensada para grandes agregados de población, se convierte en un reto abordar metodológicamente los micro espacios, pero dada la estabilidad y niveles alcanzados por los indicadores de las variables demográficas en estos tiempos, y la necesidad de abrir un abanico de opciones en cuanto a la utilidad de la ciencia para los políticos, unido a la relevancia que están ganando las acciones de desarrollo a pequeñas escalas y la necesidad de búsqueda de gobernabilidad y sustentabilidad en estos micromundos es un reto impostergable para la Demografía hoy. Un enfoque de indagación cualitativo podría apoyar o ser una de las posibles maneras de indagar y encontrar explicaciones en los pequeños espacios.

\section{La emergencia de lo cualitativo en la Demografía}

Un breve recorrido por la evolución demográfica de la población, y por su tratamiento teórico y principales problemáticas, llama la atención sobre la necesidad de la modificación también de los postulados epistemológicos y metodológicos que dicha ciencia está requiriendo. 
Se ha de partir de que durante muchos años, ha sido tradicional el estudio de las variables demográficas y sus comportamientos, asociados al cálculo de los indicadores clásicos, obteniendo datos cuantitativos para la realización de análisis muy apegados a la matemática, la estadística y otras ciencias afines. Esta información aportó durante décadas los elementos necesarios para comprender la dinámica de la población y sus comportamientos asociados, sobre todo en la medida en que estaban respondiendo a las teorías que explicaban dichos cambios.

Sin embargo, todo parece indicar que se está imponiendo la necesidad de apelar a recursos metodológicos de indagación que trasciendan lo netamente descriptivo y que apunten hacia la explicación de los acontecimientos. Se necesita acudir a enfoques metodológicos que favorezcan la recogida y tratamiento de un cuerpo de información cualitativa, partiendo de la necesidad de una perspectiva desde la cual sea posible una comprensión más amplia, completa y global del universo a estudiar.

Es que la línea del saber metodológico, en las ciencias sociales, ha transitado desde una 'epistemología de la divergencia' a una 'epistemología de la convergencia'. Se está transitando entre los epistemólogos actuales desde el positivismo a ultranza, donde se resalta a todo costo el valor de lo cuantitativo y un cualitativismo extremo que rechaza la más ínfima cuantificación, hacia un momento de convergencia donde se sopese el valor exacto de las concepciones teóricas, metodológicas y epistemológicas de los dos enfoques y se fusionen hacia la búsqueda del conocimiento más próximo a la realidad social.

Evidentemente, hablar de esta convergencia no es coincidente con las posiciones eclecticistas que en ocasiones se suelen encontrar. Por convergencia se entendería en este caso el contacto entre diferentes paradigmas para una mejor comprensión de la realidad. En el eclecticismo se seleccionan métodos diversos sin base cognoscitiva ni filosófica alguna. La convergencia se refiere a la integración de modelos y aproximaciones, pero desde postulados previamente definidos.
Vale por tanto resaltar que la pluralidad de prismas y perspectivas para abordar un fenómeno en estudio desde divisas como son la flexibilidad y la apertura en el proceso investigativo, son premisas de estos postulados, teniendo como presupuesto esencial el conocimiento exhaustivo del objeto a estudiar. Se supone una constante, la renovación de los instrumentos en la medida en que el mismo objetivo lo demanda, considerado que el gran fin del instrumento es buscar nuevos niveles de información que aún no han sido indagados.

Sin lugar a dudas, la realidad demográfica del mundo y las aproximaciones teóricas que describen e interpretan dicha movilidad, precisan una mirada bajo estos prismas.

Dicha incorporación no significa un abandono del arsenal técnico que acompaña a la Demografía desde su surgimiento y que por lo demás ha venido desarrollándose y modernizándose, también de manera acelerada, sino que esta se ha de interpretar desde la necesidad de acudir a paradigmas y enfoques epistemológicos cualitativos, entendiendo lo epistemológico como construcción de los procesos del conocimiento, en los cuales bajo una mirada integradora sea posible fusionar lo cuantitativo y lo cualitativo, o seleccionarlo en función de los objetivos concretos en cada investigación.

No obstante, en estas circunstancias se mantendría un cuestionamiento interesante: ¿qué ventajas le puede ofrecer a la Demografía el empleo específicamente de la metodología cualitativa?

Ante todo, vale destacar que las técnicas asociadas a este tipo de metodología ofrecen la posibilidad de indagar en la comprensión de los fenómenos desde lo individual, de modo que posibilita la obtención de información a un nivel menos agregado. Paralelamente, dichas técnicas tienen la peculiaridad de poder aportar información amplia y detallada sobre los comportamientos de las personas, y así profundizar en descripciones e interpretaciones de los mismos. Permite encontrar, además, la explicación de las variables asociadas a factores socioculturales, coyunturales y particulares. Así, desde lo particular, es 
posible ampliar y esclarecer las regularidades más generales. Finalmente, brindan la posibilidad de establecer hipótesis diversas acerca de la explicación de los fenómenos de la población en estudio.

Por supuesto que esta incorporación posibilita la fusión de estrategias metodológicas de corte cuantitativo y cualitativo, pero no de manera acrítica ni sumergida dentro de cada ciencia, sino desde la delimitación de problemáticas de investigación para cuya solución se precise acudir a esos enfoques. De ese mismo modo, se ha de realizar una adecuada selección de técnicas que aporten datos precisos en correspondencia con el objeto de indagación.

\section{Ejemplificando desde la fecundidad}

Tomando por ejemplo, a la variable fecundidad como objeto de estudio, sería interesante comprender qué le aportaría a su conocimiento un enfoque indagatorio de corte cualitativo.

En muchas regiones y países, esta variable se ha mantenido en niveles muy bajos y tampoco han variado de manera sustantiva el rol de sus determinantes próximos ya conocidos -anticoncepción, aborto, nupcialidad. En esta situación, se necesita transitar hacia la búsqueda de elementos explicativos del funcionamiento de estos determinantes próximos sobre la variable.

Para ello, además de ser preciso que, desde las técnicas de análisis clásico, se indague sobre las regularidades del nivel agregado o macro social, delimitando los comportamientos según los diferenciales de la fecundidad, es imprescindible encontrar argumentaciones que expliquen el nivel de los indicadores, que permitan profundizar en la búsqueda de explicaciones y formulación de hipótesis sobre los factores o elementos que condicionan dichos comportamientos. Un acercamiento a ello, transitando hacia el nivel individual, sería una manera de hacerlo, tomando en cuenta que es al final el propio individuo el protagonista de los eventos que conducen en última instancia al nacimiento de un nuevo ser.

Las variables demográficas son el reflejo a nivel social de la actuación de las poblaciones. Una vez que es evidente la necesidad de profundizar en su estudio, el camino para hacerlo a través de la comprensión de las actuaciones individuales se convierte en una alternativa de análisis. Una comprensión por ende, de la fecundidad y sus determinantes, precisa tomar en cuenta las interacciones que se producen en el interior de la realidad social. Es decir, se ha de valorar la relación entre el individuo y la sociedad, y el rol de dicha interacción en las decisiones de los individuos.

Un enfoque de este tipo podría partir de la delimitación de tres niveles en el entramado social. Entiéndase como tales, un nivel macro social, en el que se ubicaría a la sociedad y un nivel micro social que le correspondería al individuo. Entre estos dos extremos, estará mediando un nivel intermedio que podría denominarse instancia meso social y que a través de ella llegará la incidencia de lo social al individuo y su comportamiento. El rol de estos niveles intermedios es justamente actuar como canalizadores de la incidencia de lo social sobre el hombre.

Esta aproximación esquemática permitiría ubicar en cada uno de estos tres niveles, los factores y determinantes que podrían explicar las decisiones asociadas al comportamiento reproductivo de las personas. Es preciso señalar que esta esquematización deviene en un recorte metodológico de la realidad que es mucho más amplia y rica y con mayor número de interrelaciones que implican al resto de las variables demográficas.

Partiendo desde el nivel macro social, en él es preciso ubicar los factores de orden económico, social y cultural en una sociedad, entendiendo como tal, desde el sistema socioeconómico imperante hasta las particularidades del entramado sociocultural. Obviamente, esta construcción cultural está delimitada por patrones y normas socialmente aceptados históricamente.

Este punto de inicio, estará determinando la manera en que funcionarán las instituciones formales de una sociedad, que devendrán en educadoras, normadoras y también proveedoras de servicios a sus ciudadanos. Es imprescindible en estos 
tiempos interrelacionar las propias instituciones con los mecanismos de difusión a ellas vinculados.

La información, en tanto que es comunicación, resulta un eslabón esencial en la cadena de influencias sobre las conductas y decisiones individuales. De hecho, estará llegando al individuo aún cuando este no la busque.

Existen algunas teorías que han insistido en el rol de la difusión y la interacción social sobre el descenso de la fecundidad en regiones poco desarrolladas (BONGAARTS; WATKINS, 1996). En este caso, con toda veracidad se ha trascendido de los propios medios de difusión oficiales, asociados a instituciones, subrayando el rol de las redes informales de comunicaciones, tanto horizontales como verticales. Esta es otra información que recibe e intercambia el individuo, en sus grupos formales e informales de pertenencia y que también estará influyendo sobre las decisiones individuales.

Así, son evaluadas dentro de la interacción social tres categorías:

- la información y las ideas;

- la evaluación;

- la influencia social.

\section{Esquema}

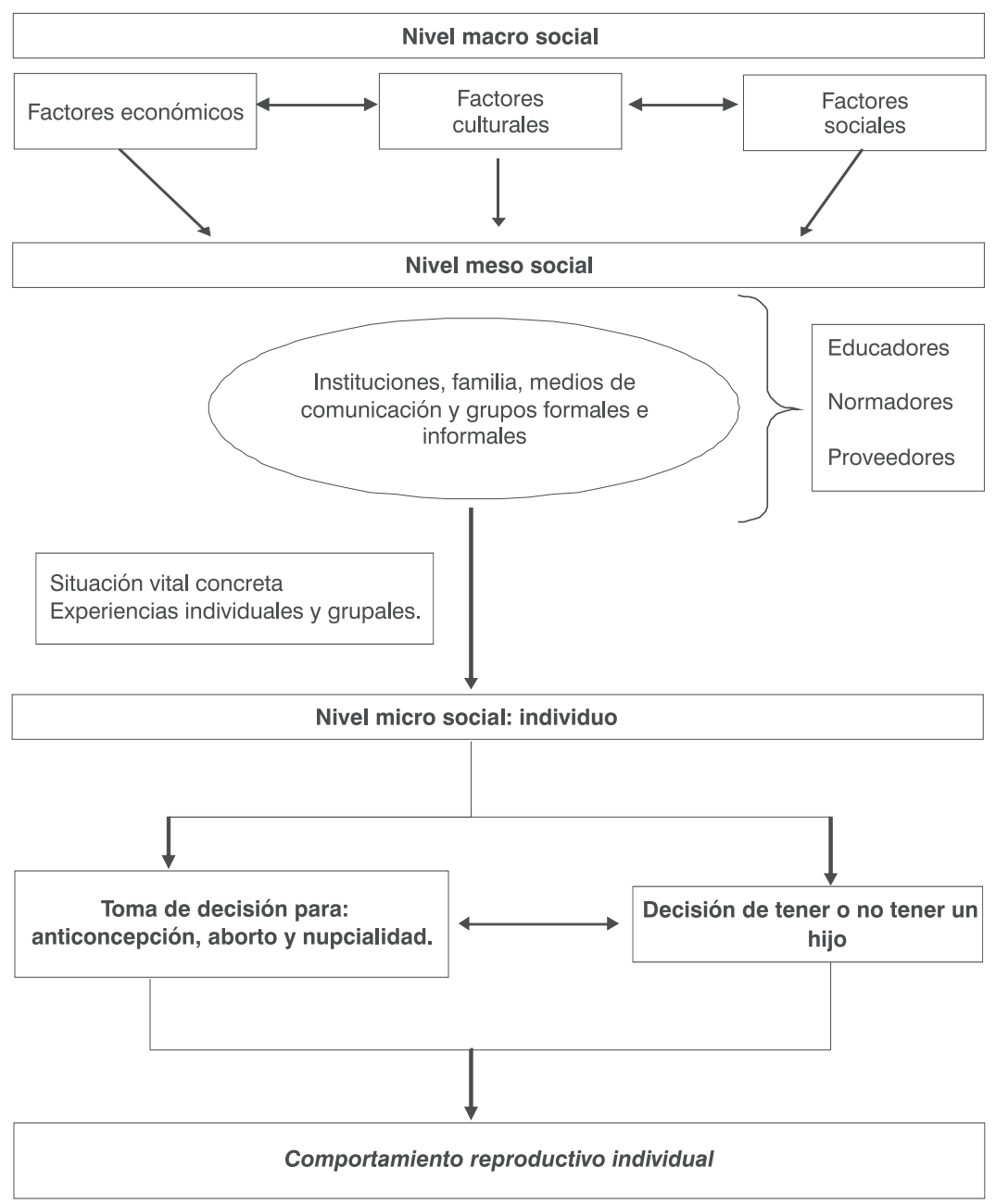


En este sentido, la información que se transmite por los diferentes canales unido a las ideas que se manejan socialmente sobre la reproducción humana, estarán legitimando e ilegitimando conductas reproductivas e incidirán de modo directo sobre los niveles de la fecundidad. Por otra parte, la evaluación que se haga de dicha información e ideas estará aportando una interpretación de las mismas, y será entonces relevante la fuente evaluadora y el significado del contexto y las circunstancias concretas en que son evaluadas. Finalmente, la influencia social se refiere al efecto que sobre el comportamiento tienen las percepciones individuales y los puntos de vista de los otros, estableciéndose los cánones de aprobación y desaprobación social y los canales para dicha interacción (BONGAARTS; WATKINS, 1996).

En el nivel meso social se está normando y regulando el funcionamiento social, oficial e informal, asociado a la reproducción. En tanto, resultan bien importantes sus funciones como proveedoras de servicios, entiéndase servicios de salud, de asistencia y a la par son estas instituciones las responsables de proporcionar educación.

Entre estas agrupaciones a las que se integra el individuo, se destaca la familia de pertenencia, cuyas normas y valores son transmitidos a sus miembros y la que en general funciona como un fuerte referente de comportamiento para él. Ahora, esta familia no es ni por mucho estática y se estará modificando en la misma medida en que recibe las influencias del nivel macro social.

Por último, en esta escala se encuentra el individuo, que resulta, como eslabón final, ser el tomador de las decisiones, pero siempre lo hará a partir de su situación vital concreta. Es decir, de su nivel educacional, inserción en el mercado laboral, situación económica, proyectos de vida etc. A la par, se estarán integrando de igual modo, las experiencias personales y las transmitidas por los diferentes grupos a los que él pertenece. Esta situación del individuo va a delimitar su actitud ante la información, quien se moverá entre los extremos de recepción acrítica y pasiva hasta la búsqueda activa y crítica de aquella información relevante.
Finalmente, esto determinará la interrelación del individuo con los llamados determinantes próximos de la fecundidad que tienen su origen en las variables intermedias de la fecundidad enunciadas por Davis y Blake (1967). En esta teoría, se divide el proceso reproductivo en 3 etapas: el coito, la concepción y la gestación y el parto. Se identifican en correspondencia once variables intermedias entre el proceso biológico y los factores económicos, sociales y culturales. En su esquema señalan:

- factores que afectan la exposición al coito (variables del coito);

- factores que afectan el riesgo de concebir (variables de la concepción);

- factores que afectan la gestación y el éxito del parto (variables de la gestación).

Estas son, a saber, "las variables intermedias a través de las cuales debe actuar cualquier factor social que influya sobre el nivel de la fecundidad" (DAVIS; BLAKE, 1967, p.157).

En este desarrollo teórico los autores ponen de relieve este conjunto de factores a través de los cuales llega siempre la influencia de lo social sobre el individuo. De hecho, ellos se interesan por diferenciar las regiones "subdesarrolladas" de las "urbanoindustriales", clarificando que diferentes combinaciones de estas variables pueden arrojar niveles similares de fecundidad.

La intención en el esquema anteriormente propuesto se refiere a comprender algunos elementos que desde lo social están incidiendo en la actuación de los determinantes que inexorablemente definirán la fecundidad. Ahora, la proposición parte en este caso de decidir que estos factores sociales condicionan la variable intermedia solo a través de la actuación concreta del individuo y, por ello, este tomará sus decisiones con relación a la anticoncepción, el aborto o la nupcialidad. La intención es complementar el esquema ya propuesto por Davis y Blake, ampliándolo hacia las interrelaciones entre lo social y lo individual.

Posteriormente Bongaarts (1982) elabora un modelo donde relaciona y operacionaliza de manera empírica dichas 
variables intermedias. De esta forma, señala la anticoncepción, la práctica del aborto inducido, la infertilidad por lactancia post parto y la nupcialidad o uniones como "determinantes próximos de la fecundidad", porque ejercen su efecto directamente sobre la fecundidad, sin interferencia posterior de otros factores.

Es evidente que un acercamiento de este orden a la variable fecundidad, precisa para la indagación de un entramado epistemológico de corte cualitativo que vaya a la búsqueda de los elementos constitutivos catalizadores o no del proceso de conformación de las decisiones individuales.

\section{Rumbos de la investigación demográfica}

El quehacer demográfico actual se encuentra entonces ante dos grandes retos que, lógicamente están recogiendo diversas particularidades desde su perspectiva interna. El primero es continuar describiendo y analizando lo que está ocurriendo en términos de los comportamientos reproductivos, la mortalidad y la movilidad de las poblaciones, mientras que el segundo consiste en proseguir en el desarrollo de

\section{Referencias}

BONGAARTS, J.; WATKINS, S. C. Social interactions and contemporary fertility transitions. Population and Development Review, v. 22, n. 4, Dec. 1996.

BURSH, T. K. Demography in a new key: a theory of population theory. Demography Research, v. 9, December 2003.

CALDWELL, J. C. Toward a restatement of demographic transition theory. Population and Development Review, v. 2, September/ December 1976.

CANALES, A.; LERNER, S. Desafíos teórico metodológicos en los estudios de población en el inicio del nuevo milenio. El Colegio de México, Universidad de Guadalajara, Sociedad Mexicana de Demografía, 2003. teorías que permitan no sólo explicar estos procesos una vez producidos, sino pronosticar conductas futuras y consecuencias de los fenómenos en estudio. Cada vez más es responsabilidad de la ciencia social y, por supuesto, también de la Demografía, el proponer insumos a políticos y tomadores de decisión para la planificación de acciones y el trazado de políticas.

Para todo ello, el posicionamiento epistemológico cualitativo brinda herramientas y un modo de hacer, constructivista y explicativo, que partiendo de los elementos ya señalados, aportará en gran medida soluciones al cumplimiento de este rol de la ciencia demográfica.

Finalmente, y no por ello menos importante la apertura a la interdisciplina, tanto desde lo teórico como desde lo metodológico, es hoy para la ciencia social y para la Demografía en particular, "no sólo una opción metodológica, sino una exigencia epistemológica en términos de construir una estrategia de investigación que permita la apertura a otra racionalidad para estudiar lo humano(...) la interdisciplina nos permite reposicionar la demografía frente a la sociedad contemporánea" (CANALES; LERNER, 2003, p. 20).

\section{CELADE-UIECP Diccionario demográfico multilingüe de la Unión Internacional para el Estudio Científico de la Población, 1985.}

COLEMAN, D. Population prospects and problems in Europe. Genus, special edition, v. LXI, n. 3-4 July-December 2005.

DAVIS, K.; BLAKE, J. La estructura social y la fecundidad. Un sistema analítico. Factores sociológicos de la fecundidad. Celade, Colmex, 1967.

GRAWITZ, M. Métodos y técnicas de las Ciencias Sociales. Barcelona-México: Editia Mexicana, Tomo I, 1984.

GONZÁLEZ, F. La investigación cualitativa en Psicología. Rumbos y desafíos. Sao Paulo: Educ, 1999. 
HERNÁNDEZ, R. EI proceso de la revolución demográfica en Cuba. Mayo. CEDEM, Universidad de La Habana, 1986.

KNODEL, J. A case for nonanthropological qualitative methods for demographers. Population and Development Review, v. 23, n. 4, December 1997.

LESTHAEGHE, R. The second demographic transition in Western countries. An interpretation. Brussels, Interuniversity program in Demography, 1991 (Working paper, 199-2).

LESTHAEGHE, R.; WILLEMS, P. Is low fertility a temporary phenomenon in the European Union? Population and Development Review, v. 25, n. 2, June 1999.

MAKLOUF, C. Qualitative methods: a key to a better understanding of demographic behavior? Population and Development Review, v. 23, n. 4, December 1997.

POURTOIS, P.; DESMET, H. Epistemología e investigación en Ciencias Humanas. Barcelona: Herdes, 1992.

PRESTON, S. The next fifteen years in demography analysis. In: TAEUBER, K. et al.

\section{Studies in population social demography.} Wisconsin: Academic Press, 1978.

RANDALL, S.; KOPPENHAVER, T. Qualitative data in demography: the sound of silence and other problems. Demographic Research, v. 9, October 2003.

RODRÍGUEZ, G. La comunidad: un contexto novedoso para estudiar la población. Novedades de población I. Cedem, 1999.

VAN DE KAA, D. J. Europe's second demographic transition. Population Bulletin, v. 42, n. 1, March 1987.

Postmodern fertility preferences: from changing value orientation to a new behaviour. Global Fertility Transition. Population and Development Review, supplement to volume 27, 2001.

The idea of a second demographic

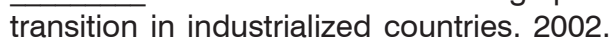
En: <www.ipss.go.jp/webj-ad/WebJournal. files/ population/2003_4/Kaa.pdf $>$.

WELTI, C. et al. Demografía I y II. Prolap. Instituto de Investigaciones Sociales de la Unam, 1997.

\section{Resumo}

Metodologia qualitativa na demografia: uma proposta a partir da fecundidade

A pertinência e a validade de incorporar o enfoque metodológico qualitativo às pesquisas demográficas constituem o objeto principal deste trabalho. Inicialmente é feita uma análise dos conceitos epistemológicos e metodológicos para a abordagem da população a partir da Demografia, repassando o objeto de estudo da ciência demográfica. A seguir, apresenta-se um breve histórico da evolução da população e das importantes teorias que acompanharam, descreveram e explicaram este processo. Assim, são trazidos elementos que validam a emergência do qualitativo para as indagações nesta ciência. Tomando-se, por exemplo, a variável fecundidade como objeto de estudo, propõe-se aplicar um enfoque teórico a partir da delimitação de três níveis: um macrossocial, em que estaria a sociedade; um nível individual; e, entre esses dois extremos, um nível intermediário, que poderia ser chamado de instância mesossocial, pela qual se chegará à incidência do social sobre o indivíduo e seu comportamento, validando, desse modo, a pertinência de abordagens qualitativas e mistas para explicar os comportamentos reprodutivos. Finalmente, conclui-se que o posicionamento epistemológico qualitativo oferece ferramentas e um modo de operar construtivista e explicativo que contribuem para o papel explicativo, preditivo e propositivo da ciência demográfica.

Palavras-chaves: Metodologia qualitativa. Demografia. Fecundidade. 


\begin{abstract}
Qualitative methodology in demography: a proposal based on fertility

The relevance and validity of incorporating qualitative methodological approach to demographic research are the subject of this paper. We begin with a review of the epistemological and methodological concepts for approaching the study of populations based on Demography, thus reviewing Demographic Science's object of study. We then present a brief history of population evolution and the major theories that followed, described and explained this process. Thus, we bring elements that validate the emergence of the qualitative to the various questions within this science. Taking, as an example, the fertility variable as an object of study, we propose a theoretical approach to delineate three levels: the macrosocial level, where society is; the individual level; and, between these two extremes, an intermediate level, which could be called the mesosocial instance, by which we arrive at the impact of the social on individuals and their behaviors, thus validating the relevance of qualitative and mixed approaches to explain reproductive behaviors. Finally, we conclude that the qualitative epistemological positioning offers tools and a constructive modus operandi and can contribute to the explanatory, predictive, and propositional roles of the demographic science.
\end{abstract}

Keywords: Qualitative methodology. Demography. Fertility.

Recebido para publicação em 26/10/2010

Aceito para publicação em 07/05/2011 